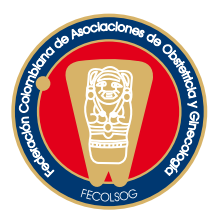

INVESTIGACIÓN ORIGINAL

\title{
LA ALTITUD COMO FACTOR DE RIESGO PARA DEFECTOS DEL TUBO NEURAL (DTN)
}

\section{Altitude as a risk factor for neural tube defects (NTD)}

Wilmar Saldarriaga, M.D., MSc.*, Gonzalo Blanco-Tamayo**, Diego Fernando Bravo-López**, Andrés Manuel Díaz-Hung**, Andrés Fandiño-Losada, M.D., MSc.***, Carolina Isaza, M.D.****

Recibido: febrero 16/07 - Revisado: agosto 13/07 - Aceptado: septiembre 11/07

\section{RESUIMEN}

Objetivo: determinar si la altura es un factor de riesgo para defectos del tubo neural (DTN) al comparar las prevalencias de defectos del tubo neural entre hospitales que utilizan la metodología del Estudio colaborativo latinoamericano de malformaciones congénitas (ECLAMC) en las ciudades de Cali y Bogotá.

Metodología: se realizó un estudio transversal haciendo uso de la base de datos del ECLAMC del Hospital Universitario del Valle (HUV) de la ciudad de Cali; Hospital Simón Bolívar, Clínica Emmanuel, Hospital Universitario San Ignacio y Clínica David Restrepo de la ciudad de Bogotá. La población en estudio fue la de los recién nacidos en dichas instituciones entre febrero del 2004 y marzo del 2005. Se estimó la razón de prevalencias entre las ciudades.

\footnotetext{
* Ginecólogo y obstetra, Maestría en Embriología y Genética, Profesor departamentos de Morfología, Ginecología y Obstetricia, Escuela de Ciencias Básicas y de Medicina, Facultad de Salud, Universidad del Valle, Hospital Universitario del Valle. Fundación Valle del Lili, Cali. Correspondencia: Calle 4ta B, No. 36 - 00. Edificio 116 espacio 4. Laboratorio de citogenética. Universidad del Valle. Correo electrónico: wsaldarriaga0608@yahoo.com

** Estudiante de VI semestre de Medicina y Cirugía, Escuela de Medicina, Facultad de Salud, Universidad del Valle, Cali. *** Maestría en Epidemiología. Investigador CISALVA (Universidad del Valle). Consultor Ministerio de la Protección Social. **** Médico y cirujano de la Universidad del Valle. Maestría en Ciencias Básicas Médicas. Vicerrectora de Investigaciones. Profesora titular del Departamento de Morfología de la Universidad del Valle, Cali.
}

Resultados: en el HUV de Cali se registraron 7.201 nacimientos entre febrero 2004 y marzo del 2005, dentro de los cuales se identificaron 21 casos de recién nacidos con DTN, para una prevalencia de 29,2 por cada 10.000 nacidos. En Bogotá la suma total de nacimientos registrados en las cuatro instituciones, fue de 15.239, y se identificaron 9 recién nacidos con DTN, para una prevalencia de seis por cada 10.000 nacidos. Se observó una diferencia estadísticamente significativa en general, para todos los DTN (RP: 4,94; 95\% IC: $2,26-10,78)$, siendo marcada para anencefalia (RP: 8,46; 95\% IC: 1,80-39,85) y para espina bífida (RP: 3,81; 95\% IC: 1,28-11,36).

Conclusiones: la prevalencia de los DTN es mayor en ciudades con altitud menor de $2.000 \mathrm{~m}$ sobre el nivel del mar, hallazgo acorde con lo reportado en la literatura.

Palabras clave: malformación congénita, defectos tubo neural, altitud.

\section{SUMMARY}

Objective: determining whether altitude is a risk factor for neural tube defects by comparing NTD prevalence amongst hospitals using the ECLAMC (The Latin-American collaborative study of congenital malformations) methodology in the cities of Cali and Bogotá in Colombia. 
Methodology: this was a cross-sectional study using ECLAMC databases from the Valle Teaching Hospital in Cali and Hospital Simón Bolívar, Clinica Emmanuel, Hospital Universitario San Ignacio and Clinica David Restrepo in Bogotá. Newborns delivered at these institutions between February 2004 and March 2005 were included in the study. Prevalence ratio was estimated.

Results: 7,201 births were registered at the Valle Teaching Hospital between February 2004 and March 2005 and 21 cases of newborns with NTD were identified (29.2/10,000 newborn prevalence). 15,239 births were registered for the same period at the four institutions in Bogotá; 9 newborns having NTD were identified (6/10,000 live newborn prevalence). A statistically significant difference was observed for all forms of NTD (RR: 4.94; 95\%CI: 2.26-10.78), markedly so for anencephaly (RR: 8.46; 95\%CI: 1.80-39.85) and spina bifida (RR: 3.81; 95\% CI: 1.28-11.36).

Conclusions: NTD prevalence was greater in cities lower than 2,000 metres above sea level, as previously reported in the literature.

Key words: congenital malformation, neural tube defect, altitude.

\section{INTRODUCCIÓN}

La residencia en lugares de gran altitud sobre el nivel del mar, como La Paz (Bolivia), el Himalaya, y zonas del Perú, está asociada con retardo en el crecimiento prenatal y otros defectos en el desarrollo del embrión y del feto, como las malformaciones congénitas. ${ }^{1-6}$

A través del Estudio colaborativo latinoamericano de malformaciones congénitas (ECLAMC), se han reportado diferencias en las frecuencias de malformaciones congénitas (MFC), en los hijos de madres que residen en ciudades ubicadas a más de 2.000 metros sobre el nivel del mar (msnm), en comparación con los hijos de madres que residen a menor altitud. Hasta el momento no se han reportado hallazgos similares en Colombia. Sin embargo, el papel actual de la altitud en la etiología de las malformaciones congénitas es todavía desconocido. ${ }^{4}$
El ECLAMC es un programa de investigación clínica y epidemiológica que opera en hospitales latinoamericanos, identificando recién nacidos con MFC, a quienes se les diligencia un formulario con 80 variables. Igualmente, se seleccionan controles para llenar el mismo formulario. De esta forma, se pueden establecer factores de riesgo asociados a las MFC y observar la prevalencia de las mismas al momento del parto.?

Castilla y colaboradores publicaron en 1999 un estudio a partir del análisis de la base de datos del ECLAMC, comparando la prevalencia de diferentes tipos de MFC en ciudades con altitud mayor a 2.000 msnm y ciudades con altitud menor a $2.000 \mathrm{msnm}$. En ese estudio se encontró que los DTN eran menos prevalentes en las ciudades más altas. El valor del riesgo relativo para anencefalia fue 0,33 (IC 99\%: $0,20-0,54)$ y para espina bífida fue 0,57 (IC 99\%: $0,37-0,78){ }^{4}$

El propósito de este estudio es determinar la diferencia en la prevalencia, al momento del parto, de los DTN entre los nacimientos de los hospitales Simón Bolívar, Clínica Emmanuel, Hospital Universitario San Ignacio y Clínica David Restrepo, de Bogotá (2.600 msnm) y el Hospital Universitario del Valle (HUV) de Cali (990 msnm). Todas las instituciones participantes desarrollan la metodología ECLAMC.

\section{MATERIALES Y MÉTODOS}

Se realizó un estudio de tipo transversal, usando las bases de datos del ECLAMC de Cali y Bogotá, las cuales hacen parte de un estudio prospectivo de casos y controles en Latinoamérica.

La población en estudio corresponde a los recién nacidos entre febrero del 2004 y marzo del 2005 en el HUV de Cali, hospital de tercer nivel de complejidad que recibe población referida de la ciudad de Cali y de la región sur occidental de Colombia; el Hospital Simón Bolívar, hospital público de tercer nivel de complejidad que atiende población con aseguramiento público; el Hospital Universitario San Ignacio centro de referencia de tercer nivel de atención; la Clínica David Restrepo y la Clínica 
Emmanuel instituciones privadas que atienden población del aseguramiento privado ubicados en Bogotá, Colombia.

Se estableció la prevalencia de DTN para cada institución de salud por ciudad, de la siguiente manera:

Se definió como caso al registro en la ficha del ECLAMC en la que se encontrara como diagnóstico anencefalia, encefalocele o defectos de espina (meningocele, mielomeningocele, mielosquisis). ${ }^{8}$

Posteriormente, se sumó el número de casos y nacimientos en las instituciones objeto del estudio por ciudad; los datos de los nacimientos se obtuvieron del informe mensual de cada centro asistencial. Estos incluyeron recién nacidos vivos y muertos con peso mayor de $500 \mathrm{~g}$.

Una vez establecida la prevalencia de DTN para el grupo de instituciones en Bogotá, se estimó la razón de prevalencias (RP) de DTN entre el HUV (Cali) y las instituciones de Bogotá, por medio de Epi Info versión 6.04d.

\section{RESULTADOS}

En el Hospital Universitario del Valle (HUV) de Cali se registraron 7.201 nacimientos entre febrero del 2004 y marzo del 2005, en estos se identificaron 21 casos de recién nacidos con defectos del tubo neural (tabla 1), para una prevalencia de 29,2 por cada 10.000 nacidos en el HUV (tabla 2).

En Bogotá, la suma total de nacimientos registrados en las cuatro instituciones participantes fue 15.239, y se identificaron dos casos de DTN en la Clínica Emmanuel, un caso en el Hospital Simón Bolívar, cinco casos en el Hospital San Ignacio y un caso en la Clínica David Restrepo, para un total de nueve recién nacidos con DTN (tabla 1), para una prevalencia en esas instituciones de seis por cada 10.000 nacidos (tabla 3).

Se observó una diferencia estadísticamente significativa en la ocurrencia de todos los tipos de DTN (RP: 4,94; 95\% IC: 2,26 - 10,78). Esta asociación es marcada para la anencefalia (RP: 8,46; 95\% IC: 1,80 - 39,85) y para la espina bífida (RP: 3,81; 95\% IC: 1,28 - 11,36) (tabla 4).

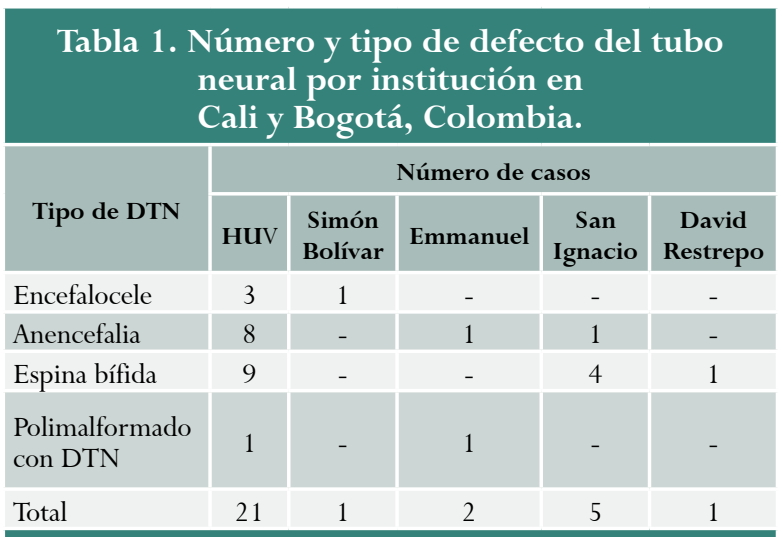

Tabla 2. Prevalencia de DTN por institución.

\begin{tabular}{|l|c|c|c|c|c|}
\hline & \multicolumn{5}{|c|}{ Prevalencia x 10.000 nacimientos } \\
\cline { 2 - 6 } Tipo de DTN & HUV & $\begin{array}{c}\text { Simón } \\
\text { Bolívar }\end{array}$ & Emmanuel & $\begin{array}{c}\text { San } \\
\text { Ignacio }\end{array}$ & $\begin{array}{c}\text { David } \\
\text { Restrepo }\end{array}$ \\
\hline Encefalocele & 4,2 & 2,4 & - & - & - \\
\hline Anencefalia & 11,1 & - & 2,8 & 2,5 & - \\
\hline Espina bífida & 12,5 & - & - & 10 & 2,8 \\
\hline $\begin{array}{l}\text { Polimalformado } \\
\text { con DTN }\end{array}$ & 1,4 & - & 2,8 & - & - \\
\hline Total & 29,2 & 2,4 & 5,6 & 12,4 & 2,8 \\
\hline
\end{tabular}

Tabla 3. Número de casos y prevalencia de DTN para Cali y Bogotá.

\begin{tabular}{|l|c|c|c|c|}
\hline & \multicolumn{2}{|c|}{ Cali } & \multicolumn{2}{c|}{ Bogotá } \\
\cline { 2 - 6 } Tipo de DTN & Casos & $\begin{array}{c}\text { Prevalencia } \\
\mathbf{x} \mathbf{1 0 . 0 0 0} \\
\text { nacimientos }\end{array}$ & Casos & $\begin{array}{c}\text { Prevalencia } \\
\mathbf{x} \mathbf{1 0 . 0 0 0} \\
\text { nacimientos }\end{array}$ \\
\hline Encefalocele & 3 & 4,2 & 1 & 0,7 \\
\hline Anencefalia & 8 & 11,1 & 2 & 1,3 \\
\hline Espina bífida & 9 & 12,5 & 5 & 3,3 \\
\hline $\begin{array}{l}\text { Polimalformado } \\
\text { con DTN }\end{array}$ & 1 & 1,4 & 1 & 0,7 \\
\hline Total & 21 & 29,2 & 9 & 6 \\
\hline
\end{tabular}

Tabla 4. Razón de prevalencias para nacimientos en altitud menor a $2.000 \mathrm{~m}$ sobre el nivel del mar.

\begin{tabular}{|l|c|c|}
\hline \multicolumn{1}{|c|}{ Tipo de DTN } & RP & IC 95\% \\
\hline Encefalocele & 6,35 & $0,66-61,03$ \\
\hline Anencefalia & 8,46 & $1,80-39,85$ \\
\hline Espina bífida & 3,81 & $1,28-11,36$ \\
\hline Polimalformado con DTN & 2,12 & $0,13-33,83$ \\
\hline Todos los DTN & 4,94 & $2,26-10,78$ \\
\hline
\end{tabular}




\section{DISCUSIÓN}

En la producción de los defectos del tubo neural intervienen múltiples factores, entre ellos genéticos, ambientales y nutricionales. En grupos poblacionales se ha encontrado una alteración en el gen que codifica la enzima metil tetrahidrofolato reductasa; un trastorno en esta genera una deficiencia en el metabolismo del ácido fólico, disminuyendo así su disponibilidad en el organismo, lo que se ha demostrado ser un factor causal de DTN. ${ }^{9-12}$

También se ha visto que en poblaciones con una dieta deficiente en ácido fólico las mujeres están predispuestas a tener hijos con DTN, por lo cual a escala mundial se han implementado políticas de salud pública con orientación hacia el mejoramiento de los niveles de ácido fólico en mujeres en edad reproductiva. En algunos países se ha recomendado el consumo de 400 microgramos diarios de ácido fólico, en mujeres en el periodo periconcepcional, como mínimo cuatro semanas antes de la concepción y 12 semanas después de la falla menstrual; y en las mujeres con el antecedente de haber tenido un hijo con DTN 5 mg día en el mismo periodo de tiempo. En otros países se fortificaron con ácido fólico ciertos alimentos de consumo masivo, como el pan, las harinas y los cereales. Todo esto con el objetivo de reducir la prevalencia de estos defectos. Con estas intervenciones se ha logrado reducir en un 70\% el número de casos de DTN. ${ }^{13-15}$

En este estudio se comparó la prevalencia de DTN en el HUV, de Cali, y los hospitales Simón Bolívar, Clínica Emmanuel, Hospital Universitario San Ignacio y Clínica David Restrepo de Bogotá. Se observó una diferencia estadísticamente significativa en la ocurrencia de todos los tipos de DTN, con mayor riesgo en la cuidad de Cali, ubicada a menor altitud. Estos hallazgos son consistentes con la literatura reportada hasta el momento. ${ }^{4}$ La prevalencia de los DTN es mayor en ciudades con altitud menor de $2.000 \mathrm{msnm}$, sin embargo, el papel fisiológico de la altitud en la aparición de DTN en niños no está claramente establecida, como sí se ha hecho en la relación con restricción del crecimiento intrauterino en las ciudades ubicadas arriba de los $2.000 \mathrm{msnm}$.
Entre las explicaciones para esta diferencia en los centros de Cali y Bogotá, es posible que se encuentre el factor nutricional ya mencionado anteriormente a escala mundial, ya que se esperaría que las dos ciudades tengan costumbres alimenticias diferentes, bien sea por el acceso a cierto grupo de productos o por el patrón cultural de alimentación. Sin embargo, serían necesarios estudios en los que se cuantifiquen los niveles de ácido fólico en la población general de dichas ciudades y a través de estos se identifique si existe un menor consumo de ácido fólico en Cali y se pudiera corroborar el papel nutricional en la etiología de los DTN en la ciudad.

Aunque no se ha podido dilucidar el papel de la altura en el desarrollo de los DTN, es claro que existe una mayor prevalencia de estos en las ciudades ubicadas en baja altitud. Sin embargo, se deberían realizar estudios similares que involucren más centros hospitalarios de diferentes ciudades colombianas, y basados en ellos, encontrar la magnitud del problema de salud publica de los DTN en Colombia y así sugerir políticas en las que idealmente se realice fortificación de alimentos con acido fólico en todo el país, o al menos se prioricen para las ciudades ubicadas debajo de los $2.000 \mathrm{~m}$ sobre el nivel del mar como Santiago de Cali. El déficit en la dieta de ácido fólico es uno de los pocos factores comprobados que aumentan la prevalencia de los DTN. ${ }^{4,13-16}$ En Colombia actualmente, se está realizando la suplementación con ácido fólico de la harina de trigo, de acuerdo con lo establecido en el Decreto 1944 de 1996, en el cual se estipula que la cantidad mínima de ácido fólico con la que se debe fortificar la harina de trigo es de 1,54 mg por kilogramo de harina. Sin embargo, es el único alimento que a la fecha se halla con suplementación, mientras otros como el arroz aún se encuentran en fase de estudios.

Por otro lado, según la encuesta nacional de la situación nutricional en Colombia (ENSIN), realizada en el 2005 por el Instituto Colombiano de Bienestar Familiar (ICBF), la ingesta diaria recomendada (RDI) de ácido fólico para la población colombiana 
es de 160 microgramos/día. Esta cifra difiere de los 400 microgramos/día recomendados para prevenir los DTN en mujeres en edad reproductiva. La harina de trigo fortificada aporta la cantidad de ácido fólico necesaria para suplir la RDI de la población en general, sin suplir las necesidades de un grupo específico (mujeres en edad reproductiva).

Además, los sistemas de vigilancia epidemiológica de las MFC aquí presentados pueden servir para medir el impacto de las intervenciones que tengan como objeto disminuir la aparición de casos de DTN.

\section{CONCLUSIÓN}

Existe una fuerte asociación estadística entre la altitud y la prevalencia de los defectos del tubo neural, se requieren estudios adicionales que relacionen otros factores de riesgo con la probabilidad de presentar estos defectos, para determinar así la existencia de una relación causal entre la altitud y los defectos del tubo neural.

\section{AGRADECIMIENTOS}

A todos los miembros del ECLAMC de Cali y Bogotá.

\section{REFERENCIAS}

1. Stinson S. The effect of high altitude on the growth of children of high socioeconomic status in Bolivia. Am J Phys Anthropol 1982;59:61-71.

2. Wiley AS. Neonatal and maternal anthropometric characteristics in high altitude population of the western Himalaya. Am J Hum Biol 1994;6:499510.

3. Wiley AS. Neonatal size and infant mortality at high altitude in the western Himalaya. Am J Phys Anthropol 1994;94:289-305.

4. Castilla EE, Lopez-Camelo J, Campaña H. Altitude as a risk factor for congenital anomalies. Am J Med Genet 1999;86:9-14.
5. Beall CM, Baker PT, Baker TS, Haas JD. The effect of high altitude on adolescent growth in Southern Peruvian Amerindians. Hum Biol 1977;49:109-24.

6. Leonard WR, De-Walt KM, Stansbury JP, McCaston MK. Growth differences between children of highland and coastal Ecuador. Am J Phys Anthropol 1995; 98:47-57.

7. Castilla E, Orioli IM. ECLAMC: The Latin-American collaborative study of congenital malformations. Community Genet 2004;7:76-94.

8. Wilson GN. Pediatric genetics: birth defect and sydromology. En: Clinical Genetics. New York, Chischester: Wiley Liss; 2000. p. 251-82.

9. Moore K, Persaud TVN. Embriología clínica. 6 ed. México DF. Mc-Graw Hill; 2001. p. 477-517.

10. Ou CY, Stevenson RE, Brown VK, Schwartz CE, Allen WP, Khoury MJ, et al. 5, 10 methylenetetrahydrofolate reductase genetic polymorphism as a risk factor for neural tube defects. Am J Med Genet 1996;63:610-4.

11. Gregory F. Genetic after of development. En: Nussbaum R, McInnes R, Willard H. Thompson and Thompson Genetics in medicine. 6th ed. Philadelphia: W.B. Saunders Co; 2001. p. 335-57.

12. Bianchi D, Crombleholme T, D'Alton M. Management of fetal conditions diagnosed by sonography. Section A: central nervous system. En: Fetology: diagnosis and management of the fetal patient. New York: Mc Graw Hill; 2000. p. 57-190.

13. Czeizel AE. Folic acid in the prevention of neural tube defects. J Pediatr Gastroenterol Nutr 1995;20:4-16.

14. Cortés F, Mellado C, Hertrampf E, Alliende A, Castillo $S$. Frecuencia de los defectos de cierre del tubo neural en las maternidades públicas de Santiago durante el año 1999. Rev Méd Chile 2001;129:277-84.

15. Castilla EE, Lopez-Camelo JE, Paz JE, Orioli IM. Prevención primaria de los defectos congénitos. Rio de Janeiro: Editora Fiocruz; 1996.

16. Berry RJ, Li Z, Erickson JD, Li S, Moore CA, Wang H, et al. Prevention of neural-tube defects with folic acid in China. China-U.S. collaborative project for neural tube defect pervention. N Engl J Med 1999;341:1485-90. 